\title{
MAGNETO-OPTIC CHARACTERIZATIONS OF SUPERLATTICES AND WEDGED SANDWICHES WITH OSCHLATORY INTERLAYER MAGNETIC COUPLING
}

\author{
S.D. Bader \\ Material Science Division, Argonne National Laboratory \\ Argonne, IL 60439 USA
}

INVITED PAPER: International Conference on the Physics of Transition Metals, (ICPTM'92), Darmstadt, Germany, July 20-24, 1992

jmc

\section{DISCLAIMER}

\begin{abstract}
This report was prepared as an account of work sponsored by an agency of the United States Government. Neither the United States Government nor any agency thereof, nor any of their employees, makes any warranty, express or implied, or assumes any legal liability or responsibility for the accuracy, completeness, or usefulness of any information, apparatus, product, or process disclosed, or represents that its use would not infringe privately owned rights. Reference herein to any specific commercial product, process, or service by trade name, trademark, manufacturer, or otherwise does not necessarily constitute or imply its endorsement, recommendation, or favoring by the United States Government or any agency thereof. The views and opinions of authors expressed herein do not necessarily state or reflect those of the United States Government or any agency thereof.
\end{abstract}

"Supported by the U.S. Department of Energy under contract \#W-31-109-ENG-38. 


\title{
MAGNETO-OPTIC CIIARACTERIZATIONS OF SUPERLATTICES ANI) WED(ZED SANDWICIIES WITI OSCIII.ATORY INTERI,AYER MAGNETIC COUPLING
}

\author{
S.D. Bader \\ Material Science Division, Argonne National Laboratory \\ Argonne, IL (6).439 USA
}

\begin{abstract}
Three examples of magnetic coupling across metallic spacer layers are considered. Fe/Nb sputtered superlattices are ebserved to have as many as five antiferromagnetic oscillations, but a weak magnetoresistive anomaly. Epitaxial triiayers of $\mathrm{Fe} / \mathrm{Mo} / \mathrm{Fe}$ grown on $\mathrm{Mo}(10(0)$ and $\mathrm{Co} / \mathrm{Cu} / \mathrm{Co}$ grown on $\mathrm{Cu}(10())$ are observed to have short- and long-period oscillations, respectively. The trilayers are grown with wedged spacer layers and characterized in-situ by means of the magneto-optic Kerr effect.
\end{abstract}

\section{Introduction}

One of the new developments in the physics of transition metals is the exploration of the magnetic coupling between films of Fe or Co separated by metallic spacer layers. These studies have led to the discoveries of both oscillatory antiferromagnetic interlayer coupling 1,2 and giant magnetoresistance. ${ }^{3}$ These new developments have revived interest in traditional theoretical concepts in transition-metal magnetism, such as oscillatory, static spin-density waves, as described by the RKKY interaction, and Fermi-surface nesting. ${ }^{4,5}$ Thus, current activities are refreshing our links with the past while they are simultaneously forging an exciting future. Today the thin-film-magnetism community is working with overlayers, bilayers, trilayers and superlattices. On the horizon we can envision the synthesis of new film configurations, including wedges of various tapers to study thickness dependences. The issue of interfacial roughness also is coming to the fore, presenting challenges in materials characterization as well as in the physics of quantum fluctuations, fractal dimensionality, and localization and interactions. In the present paper some recent experiments performed at Argonne will be highlighted involving coupled magnetic layers. Examples will be presented of both sputtered superlattices and trilayer wedges grown epitaxially in ultrahigh vacuum.

The sputtered superlattice is a medium of choice due to ease of sample preparation. Many samples can be prepared to span a range of thicknesses. Sputtered films tend to grow textured along a close-packed direction: (110) for bcc and (111) for fcc films. The superlattice configuration permits sample removal from vacuum and the use of bulk characterization techniques. Most importantly, $x$-ray diffraction permits the characterization of the atomic order within the layers, and the coherency between the layers, via scans of high-and low-angle Bragg peaks. In the future, studies of diffuse scattering away from the Bragg conditions should permit the quantification of the different types of structural disorder.

\section{Results and Discussion}

\section{I Fe/Nb Sputtered Superlattices}

The Fe/Nb superlattice system ${ }^{6}$ is highly ordered with only atomic-scale roughness, based on its $x$-ray data. SQUID magnetometry, however, demonstrates that the Fe does not retain its full moment. Presumably the interfacial Fe loses its moment due to hybridization with the $\mathrm{Nb}$. In this regard, it is interesting to recall early experiments on dilute magnetic alloys. ${ }^{7} \mathrm{Fe}$ atoms dissolved in a $\mathrm{Nb}$ host lose their moment. We see in Fig. 1 that $\mathrm{Fe}(20 \AA) / \mathrm{Nb}$ superlattices exhibit as many as five antiferromagnetic (AF) oscillations, as observed from SQUID and Kerr-effect hysteresis loops. The calipers of the loops include the squareness ratio $M_{R} / M_{S}$ of the remanent-to-saturation magnetization, and $\mathrm{H}_{\mathrm{C}} / \mathrm{H}_{\mathrm{S}}$ the coercive-to- 
switching-field rattio, where Is is the field needed to align all the magnetic layers parallel to the applied field. Magnetometry is sensitive to the emtire sample of $1-\mathrm{cm}$ dimensions, while the Kerr effect is sensitive to top few $102 \AA$ of the $1-1 \mathrm{~mm}$ region illuminated with the laser. Any detailed differences between the probes is attributed to sample inhomogeneities. Despite small differences, the observation of five AF oscillations is a notable achievement for a sputtered superlattice.

Note (Fig. 1, botton panel) that the magnetoresistance (MR) of Fe/Nb superlattices is not giant. It is only $0.3 \%$ at its maximum at room temperature $(0.5 \%$ at $4.2 \mathrm{~K})$. This points out an example - one among many - that giant $M R$ and $A F$ coupling are not necessarily prerequisite for each other. The most striking recent examples to demonstrate the importance of spin-dependent interfacial scattering are the studies of magnetic particles suspended in non-magnetic host materials. 8,9 These grainy mixtures have their magnetization directions misoriented in the absence of an applied magnetic field, and parallel aligned otherwise. Thus, they provide a similar 'switchable' environment to the layered materials of primary interest herein. Indeed, poorly layered films probably can resemble compositionally modulated arrays of grainy suspensions interleaved with non-magnetic host material.

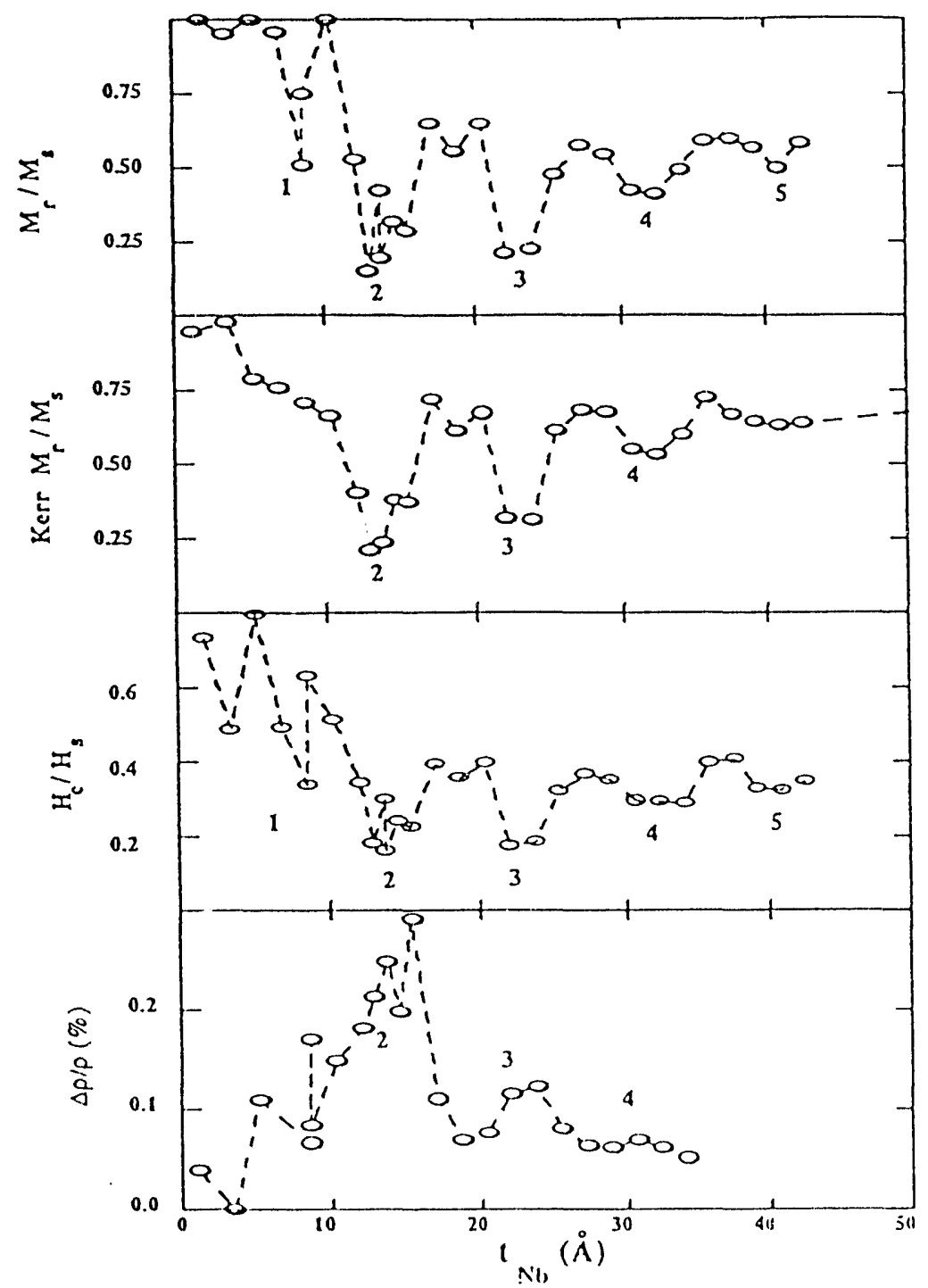

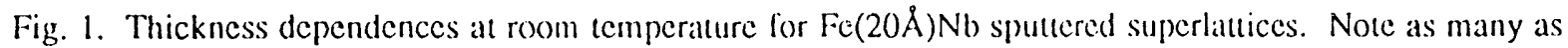
five oscillations in the antiferromagnetic coupling. Top and third panels are obtained from magnetometry.

The period of oscillation of $\sim 9 \AA$ of the AF coupling in Fig. 1 is puzzling to understand. While an $\sim 1()$ - $\AA$ value is fairly universal, independent of the nature of the metallic spacer, it 
is longer than that expecked from RKKY theory or from lemi-surace calipers. The theoretical ideals used to rationalize the 10 - $\AA$-lype period involve either aliasing or roughness to smooth out or average out, respectively, the expected short-period oscillations. ${ }^{10,11}$

\subsection{Fe/Molfe Epiexial Trilayers (irown on Mo(I0))}

It is instructive now to examine epitaxial films. The $\mathrm{Fe} / \mathrm{Nb}$ superlattice considered above is highly lattice-mismatched and has a high residual resistivity. However, it has been possible to grow epitaxial trilayers of $\mathrm{Fe} / \mathrm{Mo} / \mathrm{Fe}$ on $\mathrm{Mo}(1()())$ even though the bec lattice constant of $\mathrm{Mo}$ is $3.15 \AA$ and that of $\mathrm{Fe}$ is $2.87 \AA$. Mo is just to the right of $\mathrm{Nb}$ in the periodic table. More importantly Mo is just below $\mathrm{Cr}$, which is historically the prototype spacer layer. Mo, however, has the advantage of not itself possessing bulk moments. Thus, it serves as a simpler material than $\mathrm{Cr}$. Mo $(10)$ ) possesses its own complexity in that it has prominent nesting features in its $t w()$-dimensional Fermi surface. ${ }^{12}$ These have been implicated in the surface reconstruction of $\mathrm{Mo}(1(0))$ by driving a periodic lattice distortion. However, for $\mathrm{Mo}(100)$ to serve as the coupling medium for two magnetic layers sandwiched on either side of it, nesting features from the bulk electronic structure may be expected to play a more prominent role than these surface-derived features. But finite-size effects in ultrathin films also introduce new electronic states with wavevectors perpendicular to the plane of the film. Ortega et al. ${ }^{13}$ have observed this very clearly for Cu grown epitaxially on $\mathrm{Co}(100)$.

Bulk Mo has a low density of states at the Fermi energy $E_{F}$. Also, in bulk dilute alloys Fe retains its moment in a Mo host. Thus, there would appear to be no Mo states available near $E_{F}$ to hybridize with $F e$ states and cause $F e$ to lose its moment at the interface. By the same token, the region near $E_{F}$ is a good candidate for the appearance of surface states, since such states emerge, symmetry permitting, from within gaps in the bulk bands. This helps to explain the importance of surface states at $E_{F}$ in driving the reconstruction mentioned above.

In the Argonne studies of $\mathrm{Fe} / \mathrm{Mo} / \mathrm{Fe}$ epitaxial trilayers the Mo is grown in the shape of a wedge, tapered on one end and thick at the other, to facilitate thickness-dependent studies. ${ }^{14}$ The focused He-Ne laser beam $(\sim 0.1 \mathrm{~mm})$ scans the length of the wedge and generates

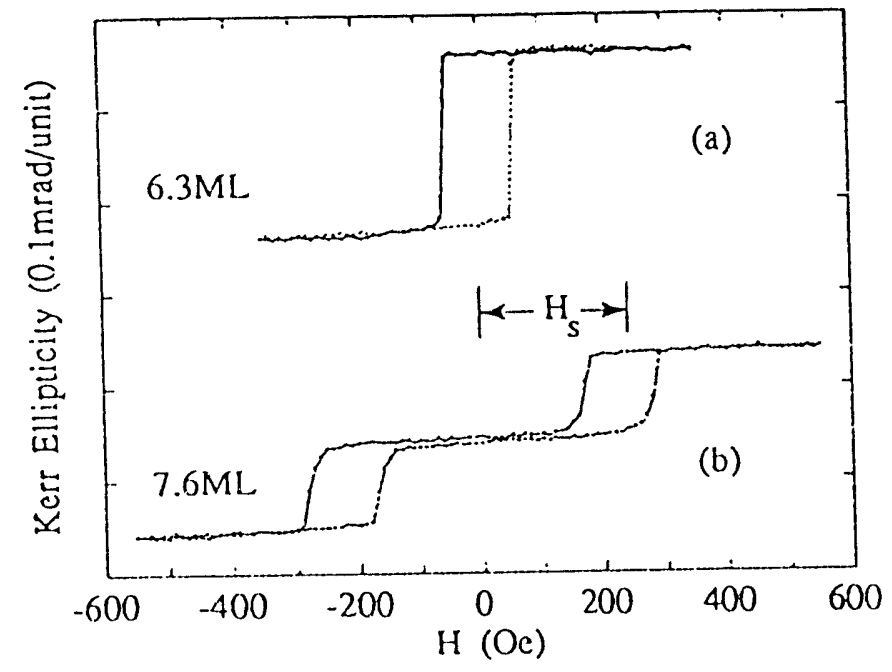

Fig. 2. Typical (a) ferromagnetic- and (b) AF-coupled hysteresis loops for an Fe(14 ML)/Mo(n ML)/Fe(14 ML) sandwich with (a) $n=6.3$ monolayers (ML) and (b) $n=7.6 \mathrm{ML}$. Note the delinition of HS.

hysteresis loops for a range of Mo thicknesses. Figure 2 shows an example of the quality of the loops. 'The features of the AF loop are sharp and striking, especially' with respect to the loops observed for sputtered films. The switching field is a well defined quantity (see Fig. 2) measured from the origin to the centroid of one of the offset hysteresis lobes. Figure 3 shows a plot of $\mathrm{H}_{\mathrm{S}}$ vs Mo thickness along the length of wedged samples. The clear trend in the data attests to the power of the wedge approach to generate materials systematics. It 


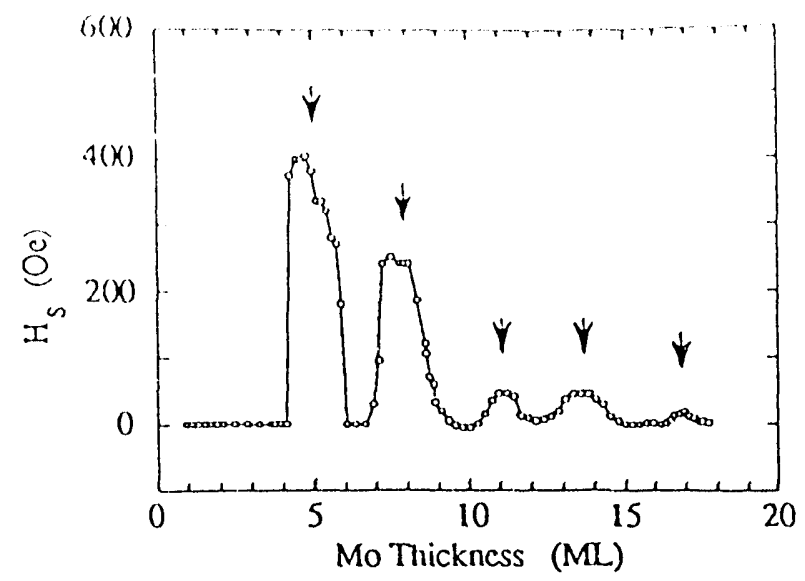

Fig. 3. Switching ficlds for $\mathrm{Fe}(14 \mathrm{ML}) / \mathrm{Mo} / \mathrm{Fe}(14 \mathrm{ML})$ wedged films showing short-period oscillations identified by arrows.

demonstrates a way to convert a molecular-beam-epitaxy machine into a high-productivity facility competitive in turnover rate with a sputtering chamber. Most importantly, Fig. 3 shows $\sim 3$-monolayer periodicity. This is a demonstration that high-quality films with reduced interfacial roughness yield periodicities in line with theoretical expectation. Sputtered Fe/Mo superlattices yield an $\sim 11-\AA$ period. 15

\subsection{Co/Cu/Co Epitaxial Trilayers Grown on Cu(100)}

$\mathrm{Co} / \mathrm{Cu} / \mathrm{Co}$ was chosen as a second trilayer system to examine at Argonne 16 because it has been studied previously magnetically and structurally, and because $\mathrm{Cu}$ represents the ultimate in simplicity for band theorists since its $d$-band is filled. In this case wedged sandwiches were chosen with equal thicknesses of $\mathrm{Co}$ on each side of the $\mathrm{Cu}$ wedge for three Co thicknesses. Sharp switching loops were observed for AF-coupled films. The $\mathrm{H}_{S}$ values were converted to surface-energy units via the expression $\mathrm{H}_{S} \mathrm{M}_{S} \mathrm{~d}_{\mathrm{C}}$, where $\mathrm{d}_{\mathrm{Co}}$ is the Colayer thickness. This provides an approximate estimate of the AF-coupling strength $\mathrm{J}_{\mathrm{AF}}$. If a film is ferromagnetically (or non-coupled) $\mathrm{H}_{\mathrm{S}}=0$ and, therefore, $\mathrm{J}_{\mathrm{AF}}=0$. Figure 4 shows the summary of the J AF data for $\mathrm{Co} / \mathrm{Cu} / \mathrm{Co}$. The period is $9.9 \AA$. Short periods were not detected even though the RHEED intensity oscillated during growth. Such RHEED oscillation are often taken as an indicator of high-quality growth. So short-period oscillations would appear to be less forgiving of roughness in the $\mathrm{Co} / \mathrm{Cu} / \mathrm{Co}$ system than for $\mathrm{Fe} / \mathrm{Mo} / \mathrm{Fe}$. Johnson, et

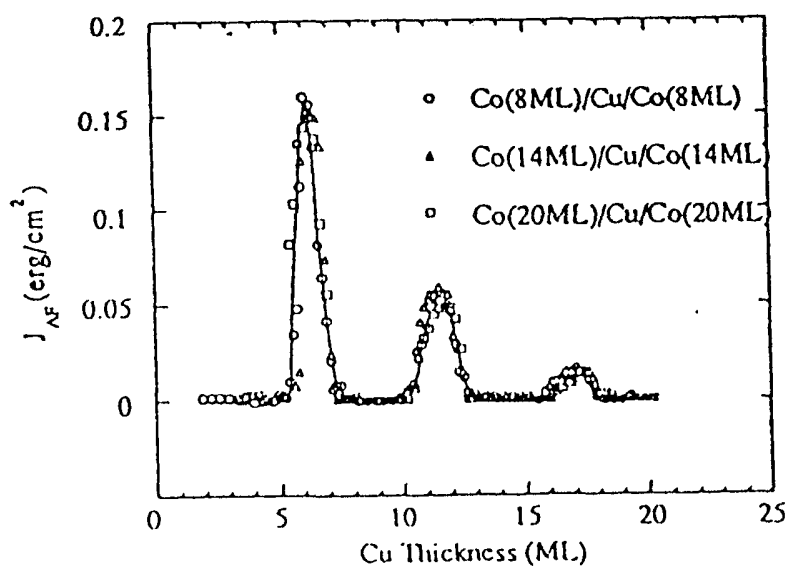

Fig. 4. Antiferromagnetic coupling strength for $\mathrm{Co} / \mathrm{Cu} / \mathrm{Co}$ sandwiches grown on $\mathrm{Cu}(\mathrm{l}(0))$ with wedged $\mathrm{Cu}$ spacer layers. The coupling strength is independent of the Co thickness. 


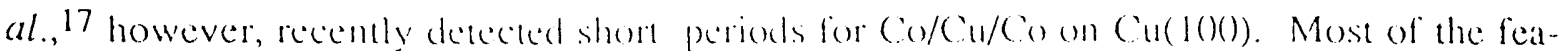
tures that they observed. hough, were of the long-period type and in exeellem agreement with the results in Fig. 4. Also, the value of the period in lign a is the same, within experimental uncertainty, as that observed in the inverse phonemission of epitaxial Cu on Co(10)) of Ref. 13. The most important leature of lige. 4 is that the three different Co-thickness curves superimpose. This means that the coupling at a lixed Cu hickness is independent of the Co thickness. Statted more direcoly, it is that the coupling occurs at the interfaces. Figure 4 actually provides an appealingly succinct statement of that basic fact. The research community has come to accept the importance of interfacial coupling and scallering in $\mathrm{Co} / \mathrm{Cu} / \mathrm{Co}$ based on a variety of different inputs, including Parkin's's studies in which a 2 - $\AA$ Co probe layer is moved in and out of the vicinity of the interface between permalloy and $\mathrm{Cu}$.

\section{Summary}

The examples presented were meant to highlight recent experiments at Argonne in coupled magnetic layers. Viewed within the context of the development of the field as a whole, the work helps point ou the relationship between structure, properties, synthesis, and theory, and suggests device applications as well. The general strategy of configuring wedged samples should certainly continue to play an important role in future studies of varied types.

\section{Acknowledgements}

The work was supported by U.S. Department of Energy, Basic Energy SciencesMaterials Sciences, under Contract \#W-31-109-ENG-38. I thank my colleagues at Argonne J.E. Mattson, J. Pearson, Z.Q. Qiu, C.H. Sowers and E.E. Fullerton for their contributions and creativity.

\section{References}

1. P. Grünberg, R. Schreiber, Y. Pang, M.B. Brodsky, and C.H. Sowers, Phys. Rev. Lett. 57 (1986) 2442.

2. S.S.P. Parkin, N. More, and K.P. Roche, Phys. Rev. Lett. 64 (1990) 2304.

3. M.N. Baibich, J. M. Broto, A. Fert, F. Nguyen Van Dau, F. Petroff, P. Etienne, G. Creuzet, A. Friederich, and J. Chazelas, Phys. Rev. Lett. 61 (1988) 2472.

4. Y. Wang, P. M. Levy, and J. L. Fry, Phys. Rev. Lett. 65 (1990) 2732.

5. P. Bruno and C. Chappert, Phys. Rev. Lett. 67 (1991) $16(1) 2$.

6. J.E. Mattson, C.H. Sowers, A. Berger, and S.D. Bader, Phys. Rev. Lett. 68 (1992) 3252.

7. A.M. Clogston, B.T. Matthias, H. Peter, H.J. Williams, E. Corenzwit, and R.C. Sherwood, Phys. Rev. 125 (1962) 541.

8. A.E. Berkowitz, J.R. Mitchell, M.J. Carey, A.P. Young, S. Zhang, F.E. Spada, F.T. Parker, A. Hutten, and G. Thomas, Phy's. Rev. Lett. 68 (1992) 3745.

9. J. Q. Xiao, J. S. Jiang, and C.L. Chien, Phys. Rev. Lett. 68 (1992) 3749.

10. D.M. Deaven, D.S. Rokhsar, and M. Johnson, Phys. Rev. B 44 (1991) 5977.

11. R. Coehoom, Phys. Rev. B 44 (1991) 9331.

12. W. Drube, D. Straub, F.J. Himpsel, P. Soukiassian, C.L. Fu, and A.J. Freeman, Phys. Rev. B 34(1986) 8989.

13. J.E. Ortega, F.J. Himpsel, G.J. Mankey, and R.F. Willis, "Quantum well states and magnetic coupling between noble metals and ferromagnets", Phys. Rev. B, submitted.

14. Z.Q. Qiu, J. Pearson, A. Berger, and S.D. Balder, Phys. Rev. Lett. 68 (1992) 1398.

15. M.E. Brubaker, J.E. Mattson, C.H. Sowers, and S.D. Bader, Appl. Phys Lett. 58 (1991) 23() 6 .

16. Z.Q. Qiu, J. Pearson, and S.D. Bader, "Oscillanory interlayer magnetic coupling of wedged $\mathrm{Co} / \mathrm{Cu} / \mathrm{Co}$ sandwiches grown on $\mathrm{Cu}(1()())$ by molecular beam epitaxy", Phys. Rev. B, submitted.

17. M.T. Johnson, S.T. Purcell, N.W.E. McGee, R. Cochoorn, J ain deStegge, and W. Hoving, Phys. Rev. Lett. 68 (199)?) 2688 .

18. S.S.P. Parkin, unpublished. 


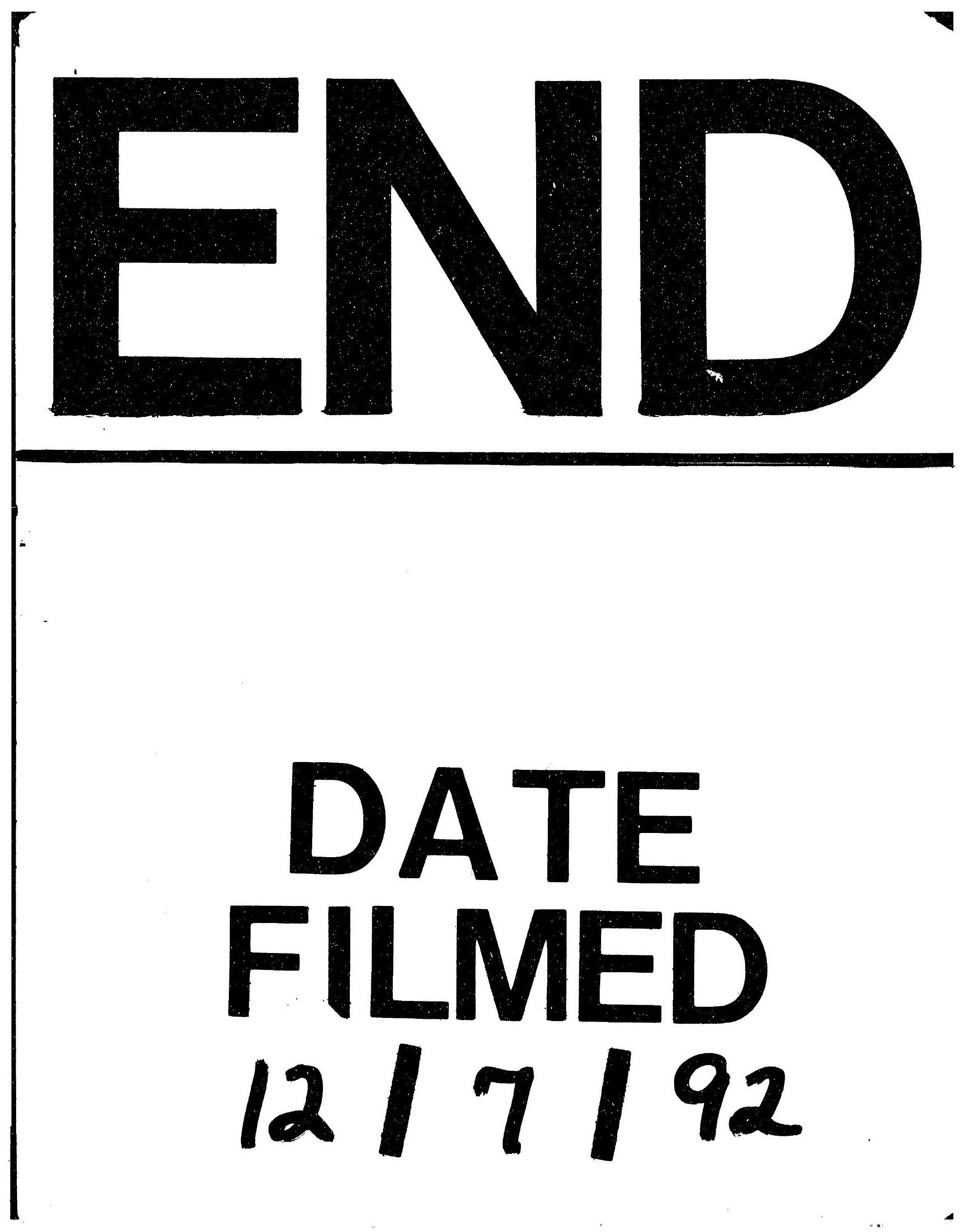


